\title{
A decomposition of Brouwer's fan theorem
}

\author{
JOSEF BERGER
}

\begin{abstract}
We introduce axioms $\mathrm{L}_{\mathrm{FAN}}$ and $\mathrm{C}_{\mathrm{FAN}}$, where the former follows from the law of excluded middle and the latter follows from the axiom of countable choice. Then we show that Brouwer's fan theorem is constructively equivalent to $\mathrm{L}_{\mathrm{FAN}}+\mathrm{C}_{\mathrm{FAN}}$. This decomposition of the fan theorem into a logical axiom and a function existence axiom contributes to the programme of constructive reverse mathematics.
\end{abstract}

2000 Mathematics Subject Classification 03F03, 03F55 (primary); 03F60 (secondary)

Keywords: fan theorem, weak König lemma, constructive mathematics, reverse mathematics

The objective of constructive reverse mathematics is to classify theorems by means of logical axioms (that means, statements which follow from the law of excluded middle) and function existence axioms (that means, statements which follow from the axiom of choice). Given a theorem $\mathrm{T}$, one aims at determining a logical axiom $\mathrm{L}_{\mathrm{T}}$ and a function existence axiom $\mathrm{C}_{T}$ such that, in the framework of a suitable base system, $\mathrm{T}$ is equivalent to $\mathrm{L}_{T}+\mathrm{C}_{T}$. A decomposition of the weak König lemma can be found in Ishihara [3]. In this paper, we provide a classification of Brouwer's fan theorem.

A suitable framework for carrying out constructive reverse mathematics is a functionbased, intuitionistic formal system, like $\mathrm{HA}^{\omega}$. In this system, if $\mathrm{A}(n)$ is equivalent to a quantifier-free formula, one may prove:

$$
\exists \alpha \in\{0,1\}^{\mathbb{N}} \forall n(\alpha(n)=0 \leftrightarrow \mathrm{A}(n)),
$$

see Troelstra and van Dalen [6, Chapter 9, Proposition 1.8].

Let $\{0,1\}^{*}$ denote the set of all finite binary sequences $u, v, w$. We write $|u|$ for the length of $u$ and $u * v$ for the concatenation of $u$ and $v$. That means, for $u=$ $(u(0), \ldots, u(l-1))$ and $v=(v(0), \ldots, v(k-1))$, we have $|u|=l$ and

$$
u * v=(u(0), \ldots, u(l-1), v(0), \ldots, v(k-1)) .
$$

If $i \in\{0,1\}$, we write $u * i$ for $u *(i)$ and $i * u$ for $(i) * u$. We use Greek letters for infinite binary sequences. We write $\bar{\alpha} n$ for $(\alpha(0), \ldots, \alpha(n-1))$. A subset A of $\{0,1\}^{*}$ is 
- detachable if there exists a function $F:\{0,1\}^{*} \rightarrow \mathbb{N}$ such that

$$
\forall u(u \in \mathrm{A} \leftrightarrow F(u)=0) ;
$$

(For example, (1) implies that, for every quantifier-free formula $\mathrm{C}(n)$, the set $\{n \mid \mathrm{C}(n)\}$ is detachable.)

- a $\Pi_{1}^{0}$-set if there exists a function $G:\{0,1\}^{*} \times \mathbb{N} \rightarrow \mathbb{N}$ such that

$$
\forall u(u \in \mathrm{A} \leftrightarrow \forall k(G(u, k)=0)) ;
$$

- closed under restriction if

$$
\forall u, v(u * v \in \mathrm{A} \rightarrow u \in \mathrm{A})
$$

- an infinite tree if

- it is detachable

- it is closed under restriction

- $\forall n \exists u(|u|=n \wedge u \in \mathrm{A})$;

- a spread if

- ()$\in \mathrm{A}$

- $\forall u(u \in \mathrm{A} \rightarrow \exists i \in\{0,1\}(u * i \in \mathrm{A}))$.

A function $\alpha$ is an infinite path of $\mathrm{A}$ if $\forall n(\bar{\alpha} n \in \mathrm{A})$. The weak König lemma reads as follows.

WKL Every infinite tree has an infinite path.

The following axiom is called the lesser limited principle of omniscience.

LLPO If a function $\alpha$ has the property

$$
\forall n, m(n \neq m \rightarrow(\alpha(n)=0 \vee \alpha(m)=0)),
$$

then

$$
\forall n(\alpha(2 n)=0) \vee \forall n(\alpha(2 n+1)=0) .
$$

Note that this axiom is a consequence of the law of excluded middle. The following characterisation of LLPO in terms of trees has also been mentioned in Berger et al. [2].

Lemma 1 The following axioms are equivalent.

(1) LLPO 
(2) If $\mathrm{T}$ is an infinite tree, then either

$$
\mathrm{T}_{0}=\{u \mid 0 * u \in \mathrm{T}\}
$$

or

$$
\mathrm{T}_{1}=\{u \mid 1 * u \in \mathrm{T}\}
$$

is an infinite tree.

Proof " $1 . \Rightarrow 2$." Assume LLPO and let T be an infinite tree. Define $\alpha$ by

$$
\alpha(2 n)=1 \stackrel{\text { def }}{\Leftrightarrow} \forall u(|u|=n \rightarrow 0 * u \notin \mathrm{T})
$$

and

$$
\alpha(2 n+1)=1 \stackrel{\text { def }}{\Leftrightarrow} \forall u(|u|=n \rightarrow 1 * u \notin \mathrm{T}) .
$$

Furthermore, define $\beta$ by

$$
\beta(n)=1 \stackrel{\text { def }}{\Leftrightarrow} \alpha(n)=1 \wedge \forall k<n(\alpha(k)=0) .
$$

By LLPO, there is an $i \in\{0,1\}$ such that $\forall n(\beta(2 n+i)=0)$. We show that $\mathrm{T}_{i}$ is an infinite tree. Suppose that there is an $m$ such that $i * w \notin \mathrm{T}$ for all $w$ with $|w|=m$. Then $\alpha(2 m+i)=1$. Since $\mathrm{T}$ is an infinite tree, we have $\forall k(\alpha(2 k+(1-i))=0)$. Therefore, there exists an $n \leq m$ such that $\beta(2 n+i)=1$. This contradiction shows that $\mathrm{T}_{i}$ is indeed an infinite tree.

"2. $\Rightarrow 1$." Assume that the function $\gamma$ has the property

$$
\forall n, m(n \neq m \rightarrow(\gamma(n)=0 \vee \gamma(m)=0)) .
$$

Define an infinite tree $\mathrm{T}$ by

$$
u \in \mathrm{T} \stackrel{\text { def }}{\Leftrightarrow} \exists i \in\{0,1\} \forall k<|u|(u(k)=i \wedge \gamma(2 k+i)=0) .
$$

If $\mathrm{T}_{i}$ is an infinite tree, then

$$
\forall n(\gamma(2 n+i)=0)
$$

The following statement is a version of the axiom of countable choice.

$\mathrm{C}_{\mathrm{WKL}} \quad$ Every $\Pi_{1}^{0}$-spread has an infinite path.

The following decomposition of WKL can be found in Ishihara [3]. We recall it, because we want to compare it with the main result of this paper, the decomposition of FAN. We even give a proof of it, because there is a slight difference between $\mathrm{C}_{\mathrm{WKL}}$ and the choice axiom used in Ishihara [3]. 


\section{Proposition 2}

$$
\mathrm{WKL} \leftrightarrow \mathrm{LLPO}+\mathrm{C}_{\mathrm{WKL}}
$$

Proof Assume WKL. First, we show LLPO by applying Lemma 1. Let T be an infinite tree. By WKL, there exists an infinite path $\alpha$ of T. Set $i=\alpha(0)$. Then

$$
\{w \mid i * w=\bar{\alpha}(|w|+1)\} \subseteq \mathrm{T}_{i} .
$$

Therefore, $\mathrm{T}_{i}$ is an infinite tree.

Next, we show $\mathrm{C}_{\mathrm{WKL}}$. To this end, let $\mathrm{A}$ be $\Pi_{1}^{0}$-spread. There is a function $G$ : $\{0,1\}^{*} \times \mathbb{N} \rightarrow \mathbb{N}$ such that

$$
\forall u(u \in \mathrm{A} \leftrightarrow \forall k(G(u, k)=0)) .
$$

Define an infinite tree $\mathrm{T}$ by

$$
u \in \mathrm{T} \stackrel{\text { def }}{\Leftrightarrow} \forall k, l \leq|u|(G(\bar{u} k, l)=0) .
$$

Then there exists an infinite path $\alpha$ of $\mathrm{T}$, which is also an infinite path of A.

Now assume both LLPO and $\mathrm{C}_{\mathrm{WKL}}$. Let $\mathrm{T}$ be an infinite tree. By LLPO and Lemma 1 we obtain that the set $\mathrm{A}$, given by

$$
u \in \mathrm{A} \stackrel{\text { def }}{\Leftrightarrow} \forall n \exists w(|w|=n \wedge u * w \in \mathrm{T}),
$$

is a spread. Therefore, by $\mathrm{C}_{\mathrm{WKL}}$, there is an infinite path $\alpha$ of $\mathrm{A}$. This function $\alpha$ is also an infinite path of $\mathrm{T}$.

A detachable subset $\mathrm{B}$ of $\{0,1\}^{*}$ is

- closed under extension if $\forall u, v(u \in \mathrm{B} \rightarrow u * v \in \mathrm{B})$;

- a bar if $\forall \alpha \exists n(\bar{\alpha} n \in \mathrm{B})$;

- a uniform bar if $\exists N \forall \alpha \exists n \leq N(\bar{\alpha} n \in \mathrm{B})$.

Brouwer's fan theorem for detachable bars reads as follows.

FAN Every bar is a uniform bar.

The following Lemma can be found in Ishihara [4].

Lemma 3 The following statements are equivalent.

- FAN

- For every bar B which is closed under extension there exists an $N$ such that $\forall u(|u|=N \rightarrow u \in \mathrm{B})$. 
- Every bar which is closed under extension is a uniform bar.

Berger and Ishihara [1] have shown that the statement 'every infinite tree with at most one infinite path has an infinite path' is constructively equivalent to FAN. See also Schwichtenberg [5] for a more formal proof of this result. This characterisation of FAN, together with Proposition 2, gives rise to the definition of the axioms $\mathrm{L}_{\mathrm{FAN}}$ and $\mathrm{C}_{\mathrm{FAN}}$.

A subset $\mathrm{A}$ of $\{0,1\}^{*}$ has at most one infinite path if

$$
\forall \alpha, \beta(\exists n(\alpha(n) \neq \beta(n)) \rightarrow \exists m(\bar{\alpha} m \notin \mathrm{A} \vee \bar{\beta} m \notin \mathrm{A})) .
$$

$\mathrm{L}_{\mathrm{FAN}}$ If $\mathrm{T}$ is an infinite tree with at most one infinite path, then either

$$
\mathrm{T}_{0}=\{u \mid 0 * u \in \mathrm{T}\}
$$

or

$$
\mathrm{T}_{1}=\{u \mid 1 * u \in \mathrm{T}\}
$$

is an infinite tree.

$\mathrm{C}_{\mathrm{FAN}}$ If $\mathrm{A}$ is a $\Pi_{1}^{0}$-spread and $\mathrm{B}$ is a bar, then there exists a $u \in \mathrm{A} \cap \mathrm{B}$.

The axioms introduced so far are related as follows.

Lemma 4 LLPO implies $\mathrm{L}_{\mathrm{FAN}}$ and $\mathrm{C}_{\mathrm{WKL}}$ implies $\mathrm{C}_{\mathrm{FAN}}$.

Proof The fact that LLPO implies $\mathrm{L}_{\mathrm{FAN}}$ follows from Lemma 1. In order to prove the second implication, assume $\mathrm{C}_{\mathrm{WKL}}$ and fix a bar $\mathrm{B}$. Assume further that $\mathrm{A}$ is a $\Pi_{1}^{0}$-spread. By $\mathrm{C}_{\mathrm{WKL}}$, there is an infinite path $\alpha$ of $\mathrm{A}$. Since B is a bar, there exists an $n$ such that $\bar{\alpha} n \in \mathrm{B}$. Thus $\bar{\alpha} n \in \mathrm{A} \cap \mathrm{B}$.

Now we are ready to prove the decomposition of FAN.

\section{Proposition 5}

$$
\mathrm{FAN} \leftrightarrow \mathrm{L}_{\mathrm{FAN}}+\mathrm{C}_{\mathrm{FAN}}
$$

Proof First we show that FAN implies $\mathrm{L}_{\mathrm{FAN}}$. Let $\mathrm{T}$ be an infinite tree with at most one infinite path. This implies

$$
\forall \alpha, \beta \exists n(0 * \bar{\alpha} n \notin \mathrm{T} \vee 1 * \bar{\beta} n \notin \mathrm{T}) .
$$

Fix $\alpha$ and define a bar B by

$$
\mathrm{B}=\{u|0 * \bar{\alpha}| u \mid \notin \mathrm{T} \vee 1 * u \notin \mathrm{T}\} .
$$


Since B is closed under extension, Lemma 3 implies the existence of an $n$ such that $\forall u(|u|=n \rightarrow u \in \mathrm{B})$. Thus we have

$$
\forall \alpha \exists n \forall \beta(0 * \bar{\alpha} n \notin \mathrm{T} \vee 1 * \bar{\beta} n \notin \mathrm{T}) .
$$

Define another bar $\mathrm{B}^{\prime}$ by

$$
\mathrm{B}^{\prime}=\{v \mid \forall \beta(0 * v \notin \mathrm{T} \vee 1 * \bar{\beta}|v| \notin \mathrm{T})\} .
$$

Again, since $\mathrm{B}^{\prime}$ is closed under extension, Lemma 3 implies the existence of an $n$ such that $\forall v\left(|v|=n \rightarrow v \in \mathrm{B}^{\prime}\right)$. We obtain

$$
\exists n \forall \alpha, \beta(0 * \bar{\alpha} n \notin \mathrm{T} \vee 1 * \bar{\beta} n \notin \mathrm{T}) .
$$

Since $\mathrm{T}$ is an infinite tree, it contains an element $u$ of length $n+1$. If $u(0)=i$, then (2) implies that

$$
\forall v(|v|=n \rightarrow(1-i) * v \notin \mathrm{T}) .
$$

But $\mathrm{T}$ is an infinite tree, therefore, $\mathrm{T}_{i}$ must be an infinite tree as well.

Next, we show that FAN implies $\mathrm{C}_{\mathrm{FAN}}$. Let $\mathrm{A}$ be a $\Pi_{1}^{0}$-spread. Then for every $n$ there is a $u$ with $|u|=n$ such that every restriction of $u$ belongs to A. Let $\mathrm{B}$ be a bar. By FAN, there is an $N$ such that

$$
\forall u(|u|=N \rightarrow \exists n \leq N(\bar{u} n \in \mathrm{B})) .
$$

Suppose that $u$ is of length $N$ and that every restriction of $u$ belongs to A. Then there is an $n \leq N$ such that $\bar{u} n \in \mathrm{A} \cap \mathrm{B}$.

Finally, we show that the combination of $\mathrm{L}_{\mathrm{FAN}}$ and $\mathrm{C}_{\mathrm{FAN}}$ implies FAN. Let $\mathrm{B}$ be a bar which is closed under extension. Define

$$
v \prec u \stackrel{\text { def }}{\Leftrightarrow}|v|<|u| \vee(|v|=|u| \wedge \exists k<|v|(\bar{v} k=\bar{u} k \wedge v(k)<u(k))) .
$$

If ()$\in \mathrm{B}$, then $\mathrm{B}$ is a uniform bar. Assume now that ()$\notin \mathrm{B}$ and define a subset $\mathrm{P}$ of $\{0,1\}^{*}$ by

$$
u \in \mathrm{P} \stackrel{\text { def }}{\Leftrightarrow} u \notin \mathrm{B} \wedge \forall v(u \prec v \rightarrow v \in \mathrm{B}) .
$$

A sequence $u$ belongs to $\mathrm{P}$ if and only if it is the largest element of $\{0,1\}^{*} \backslash \mathrm{B}$, with respect to the ordering $\prec$. Since $\mathrm{B}$ is closed under extension, we can conclude that $u \in \mathrm{P}$ if and only if

$$
u \notin \mathrm{B} \wedge \forall v(|v| \leq|u|+1 \wedge u \prec v \rightarrow v \in \mathrm{B}) .
$$

Therefore, $\mathrm{P}$ is detachable. Since $\prec$ is a total relation on $\{0,1\}^{*}$, we can conclude that $\mathrm{P}$ has at most one element, that means

$$
\forall u, v(u \neq v \rightarrow u \notin P \vee v \notin \mathrm{P}) .
$$


Furthermore, if there exists an element $u$ of $\mathrm{P}$, then every $w$ with $|w|=|u|+1$ belongs to $\mathrm{B}$, which implies that $\mathrm{B}$ is a uniform bar. Define an infinite tree $\mathrm{T}$ by

$$
u \in \mathrm{T} \stackrel{\text { def }}{\Leftrightarrow} u \notin \mathrm{B} \vee \exists n<|u|(\bar{u} n \in \mathrm{P} \wedge \forall l(n \leq l<|u| \rightarrow u(l)=0)) .
$$

Note that here we use the assumption that ()$\notin B$, because $B=\{0,1\}^{*}$ would imply that $\mathrm{T}=\emptyset$. If a sequence $u$ belongs to $\mathrm{T}$, then either it does not belong to $\mathrm{B}$ or else some proper restriction of $u$ belongs to $\mathrm{P}$. We show that $\mathrm{T}$ has at most one infinite path. Fix $\alpha$ and $\beta$ and suppose that there is an $m$ such that $\alpha(m) \neq \beta(m)$. Since B is both a bar and closed under extension, there is an $n$ such that $\bar{\alpha} n \neq \bar{\beta} n, \bar{\alpha} n \in \mathrm{B}$, and $\bar{\beta} n \in \mathrm{B}$. We will derive a contradiction from the assumption that both $\bar{\alpha} n$ and $\bar{\beta} n$ are in $\mathrm{T}$. By the definition of $\mathrm{T}$, there are $k, l<n$ such that

- $\bar{\alpha} k \in \mathrm{P}$;

- $\alpha(k)=\alpha(k+1)=\ldots=\alpha(n-1)=0$;

- $\bar{\beta} l \in \mathrm{P}$;

- $\beta(l)=\beta(l+1)=\ldots=\beta(n-1)=0$.

If $\bar{\alpha} k=\bar{\beta} l$, then $\bar{\alpha} n=\bar{\beta} n$. If $\bar{\alpha} k \neq \bar{\beta} l$, then $\bar{\alpha} k$ and $\bar{\beta} l$ are two distinct elements of P. Either case leads to a contradiction. Therefore, $\mathrm{T}$ has at most one infinite path. Define a subset $\mathrm{T}^{\prime}$ of $\mathrm{T}$ by

$$
u \in \mathrm{T}^{\prime} \stackrel{\text { def }}{\Leftrightarrow}\{w \mid u * w \in \mathrm{T}\} \text { is an infinite tree. }
$$

Since we have

$$
\forall u\left(u \in \mathrm{T}^{\prime} \leftrightarrow \forall m \exists w(|w|=m \wedge u * w \in \mathrm{T})\right),
$$

we can conclude that $T^{\prime}$ is a $\Pi_{1}^{0}$-set. Next, we show that $T^{\prime}$ is a spread. Since $T$ is an infinite tree, ()$\in \mathrm{T}^{\prime}$. Assume that $u \in \mathrm{T}^{\prime}$. Then

$$
T_{u}=\{w \mid u * w \in \mathrm{T}\}
$$

is an infinite tree. By $\mathrm{L}_{\mathrm{FAN}}$ there exists $i \in\{0,1\}$ such that $\left(T_{u}\right)_{i}$ is an infinite tree, which implies that $u * i$ is in $\mathrm{T}^{\prime}$. This concludes the proof that $\mathrm{T}^{\prime}$ is a spread. Now $\mathrm{C}_{\text {FAN }}$ yields the existence of a $u$ such that $u \in \mathrm{T}^{\prime} \cap \mathrm{B}$. Since we have $\mathrm{T}^{\prime} \subseteq \mathrm{T}$, we even obtain that $u \in \mathrm{B} \cap \mathrm{T}$. By the definition of $\mathrm{T}$, some restriction of $u$ lies in $\mathrm{P}$. Therefore, $\mathrm{B}$ is a uniform bar.

Overall, we obtain the following picture.

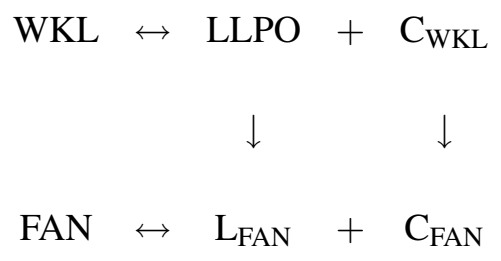


Acknowledgment While writing this paper, the author was being supported by a 'Feodor Lynen Return Fellowship', provided by the Alexander von HumboldtFoundation.

\section{References}

[1] Josef Berger, Hajime Ishihara, 'Brouwer's fan theorem and unique existence in constructive analysis.' Math. Log. Quart. 51, no. 4 (2005), 360-364; doi:10.1002/malq.200410038.

[2] Josef Berger, Hajime Ishihara, Peter Schuster, 'The Weak König Lemma, Brouwer's Fan Theorem, De Morgan's Law, and Dependent Choice', Preprint (2008.

[3] Hajime Ishihara, 'Constructive Reverse Mathematics: Compactness Properties.' In: From Sets and Types to Analysis and Topology: Towards Practicable Foundations for Constructive Mathematics (L. Crosilla and P. Schuster, eds), Oxford University Press. Oxford Logic Guides 48 (2005), 245-267.

[4] Hajime Ishihara, 'Weak König's Lemma Implies Brouwer's Fan Theorem: A Direct Proof.' Notre Dame Journal of Formal Logic 47, no 2 (2006), 249-252; doi:10.1305/ndjfl/1153858649.

[5] Helmut Schwichtenberg, 'A Direct Proof of the Equivalence between Brouwer's Fan Theorem and König's Lemma with a Uniqueness Hypothesis.' C. Calude and H. Ishihara, eds., Constructivity, Computability, and Logic. A Collection of Papers in Honour of the 60th Birthday of Douglas Bridges. Journal of Universal Computer Science 11, no. 12 (2005), 2086-2095; doi:10.3217/jucs-011-12-2086.

[6] Anne S. Troelstra, Dirk van Dalen, Constructivism in Mathematics. An Introduction. Vol. I and II. Studies in Logic and the Foundation of Mathematics, Vol. 121 and 123, North-Holland (1988).

Mathematisches Institut, Universität München

Theresienstraße 39, 80333 München, Germany

jberger@mathematik.uni-muenchen.de

http://www.mathematik.uni-muenchen.de/ jberger

Received: 30 January $2009 \quad$ Revised: 22 April 2009 